\title{
AVALIAÇÃO DA EFICIÊNCIA NUTRICIONAL DE TRÊS PROCEDÊNCIAS AUSTRALIANAS DE ACÁCIA-NEGRA (Acacia mearnsii De Wild.) ${ }^{1}$
}

\author{
Marcos Vinicius Winckler Caldeira ${ }^{2}$, Rubens Marques Rondon Neto $^{2}$ e Mauro Valdir Schumacher ${ }^{3}$
}

\begin{abstract}
RESUMO - O objetivo do presente trabalho foi avaliar a eficiência nutricional de macronutrientes $\mathrm{N}, \mathrm{P}, \mathrm{K}, \mathrm{Ca}$, $\mathrm{Mg}$ e $\mathrm{S}$ nos diferentes componentes das árvores de três procedências de acácia-negra (Acacia mearnsii De Wild.), com 2,4 anos de idade, plantadas em Butiá-RS (Brasil). As procedências selecionadas foram Lake George, Bodalla e Batemans Bay. Foi constatada variação na eficiência nutricional entre as procedências. Em ordem decrescente de eficiência de utilização dos nutrientes para produção de casca e madeira, cujos produtos são explorados dos povoamentos, obteve-se: Bodalla > Batemans Bay > Lake George. Embora a Lake George seja a procedência menos eficiente no uso dos nutrientes para formação de copa, casca e madeira, ela possui grande capacidade produtiva.
\end{abstract}

Palavras-chave: Eficiência nutricional, Acacia mearnsii e macronutrientes.

\section{EVALUATION OF THE NUTRITIONAL EFFICIENCY OF THREE AUSTRALIAN PROVENANCES OF BLACK WATTLE (Acacia mearnsii De Wild.)}

\begin{abstract}
The present study aimed to determine the nutritional efficiency of the macronutrients $N, P, K, C a$, $\mathrm{Mg}$ and $\mathrm{S}$, in the different components of trees from three provenances of black wattle (Acacia mearnsii De Wild.), 2.4 years old, planted in Butiá, RS, Brazil. The selected Australian provenances were Lake George, Bodalla and Batemans Bay. There was a wide variation in the nutritional efficiency among the provenances. In a decreasing ranking for efficiency of nutrient utilization for the production of wood and bark, products that are exploited in the even-aged forest, the following results were obtained: Bodalla > Batemans Bay > Lake George. Although Lake George is less efficient in using nutrients to produce wood, bark and crown, it has a great capacity of production.
\end{abstract}

Key words: $\quad$ Nutritional efficiency, Acacia mearnsii and macronutrients.

\section{INTRODUÇÃO}

No Brasil, a acácia-negra (Acacia mearnsii De Wild.) é plantada principalmente no Estado do Rio Grande do Sul, cobrindo aproximadamente 100.000 ha (Higa et al., 1998). O objetivo principal do reflorestamento com acácia é a extração da casca para produção de tanino, mas a madeira também é utilizada na fabricação de papel e celulose e de aglomerados. Os povoamentos, em sua grande maioria, são implantados em solos de baixa fertilidade e as práticas de uso e manejo do solo normalmente são realizadas de forma incorreta. Sob tais condições, os índices de produtividade geralmente são baixos (Dallago, 2000).

A seleção de material genético que melhor se adapte à condição de fertilidade mais baixa do solo tem sido

1 Recebido para publicação em 6.11.2001.

Aceito para publicação em 6.11.2002.

2 Eng. Florestal, Doutorando em Ciências Florestais, Centro de Ciências Florestais e da Madeira/Universidade Federal do Paraná, Av. Pref. Lothário Meissner, 3400, Jardim Botânico, 80210-170 Curitiba-PR, <caldeira@ floresta.ufpr.br>, Bolsista do CNPq; ${ }^{3}$ Eng. Florestal, Dr. Prof. Adjunto do Departamento de Ciências Florestais, Centro de Ciências Rurais/Universidade Federal de Santa Maria, Campus Universitário, Camobi, 97105-900 Santa Maria-RS. 
uma preocupação constante, sendo as espécies desejáveis aquelas com maior capacidade de absorver e utilizar os nutrientes (Morais et al., 1990). A avaliação da eficiência de utilização dos nutrientes por parte das diferentes espécies florestais, procedências e, ou, clones é um importante parâmetro para auxiliar o silvicultor no momento de optar pelo material a ser utilizado nos reflorestamentos.

O objetivo do presente estudo foi procurar indivíduos mais eficientes quanto à utilização de macronutrientes para produção de biomassa acima do solo em povoamentos de acácia-negra com 2,4 anos de idade, plantada no município de Butiá-RS.

\section{MATERIAL E MÉTODOS}

A coleta do material foi realizada em fevereiro de 1997, em três povoamentos de acácia-negra instalados em um único sítio, com 2,4 anos de idade, no espaçamento 1,7 x 3,0 m. O plantio está situado na região fisionômica natural do Estado do Rio Grande do Sul, denominada Serra do Sudeste (Escudo Rio-grandense), município de Butiá - RS, situado entre as coordenadas geográficas $30^{\circ} 07^{\prime} 12^{\prime \prime} \mathrm{S}$ e $51^{\circ} 57^{\prime} 45^{\prime \prime} \mathrm{W}$ e altitude média de $35 \mathrm{~m}$.

O clima da região é do tipo Cfa, segundo a classificação de Köppen, sendo do tipo subtropical (Moreno, 1961), com temperatura média anual de 18 a $19{ }^{\circ} \mathrm{C}$ e médias máximas e mínimas anuais de $24 \mathrm{e} 14{ }^{\circ} \mathrm{C}$, respectivamente. A precipitação média anual é de $1.400 \mathrm{~mm}$ (IPAGRO, 1989).
O solo da região pertence à Unidade de Mapeamento São Jerônimo, classificado como Argissolo VermelhoEscuro (EMBRAPA, 1999), com textura argilosa e relevo ondulado. No Quadro 1 encontram-se os resultados das análises químicas do solo onde foi estabelecido o experimento.

Para quantificação da biomassa e do conteúdo de macronutrientes acima do solo da acácia-negra, foram selecionadas nove árvores de cada procedência, sendo: Batemans Bay, New South Wales ( $35^{\circ} 15^{\prime}$ S e $150^{\circ} 15^{\prime}$ E e $20 \mathrm{~m}$ de altitude); Lake George, New South Wales ( $35^{\circ} 28^{\prime} \mathrm{S}$ e $148^{\circ} 57^{\prime}$ 'E e $700 \mathrm{~m}$ de altitude); e Bodalla, New South Wales ( $36^{\circ} 11^{\prime}$ 'S e $149^{\circ} 58^{\prime}$ E e $15 \mathrm{~m}$ de altitude). A seleção das árvores para este estudo foi a partir dos dados do inventário florestal, que permitiu agrupálas em nove classes diamétricas, tendo sido retirada uma árvore por cada classe, em cada procedência.

Na parte intermediária da copa das árvores, nos quatro pontos cardeais, foram coletados cerca de $300 \mathrm{~g}$ de folhas, para realização de análise nutricional. Os galhos foram classificados como vivos e mortos, tendo sido colhidas todas as folhas dos galhos vivos.

A massa fresca total das folhas, dos galhos vivos e mortos, da casca e da madeira do tronco das nove árvores foi determinada no campo. De cada um desses componentes foram retiradas subamostras, para aferição da massa fresca no campo. Posteriormente, cada subamostra foi acondicionada em sacos plásticos, identificada e

Quadro 1 - Características químicas do Argissolo Vermelho-Escuro, em três camadas de profundidade, dos locais de plantios das três procedências de acácia-negra, no município de Butiá-RS

Table 1 - Chemical characteristics of dark red Argisol at three depth levels in an even-aged forest of three black wattle provenances in Butiá-RS

\begin{tabular}{|c|c|c|c|c|c|c|c|c|c|c|}
\hline \multirow[t]{2}{*}{ Procedência } & $\begin{array}{c}\text { Profun- } \\
\text { didade }\end{array}$ & $\mathrm{pH} \mathrm{H} \mathrm{H}_{2} \mathrm{O}$ & M.O. & $\mathrm{P}$ & $\mathrm{K}$ & $\mathrm{Ca}$ & $\mathrm{Mg}$ & $\mathrm{Al}$ & \multirow{2}{*}{$\begin{array}{c}\text { CTC } \\
\text { Efetiva }\end{array}$} & $\begin{array}{c}\text { Saturação de } \\
\text { Bases }\end{array}$ \\
\hline & $(\mathrm{cm})$ & $(1: 1)$ & $(\mathrm{g} / \mathrm{kg})$ & \multicolumn{2}{|c|}{$\left(\mathrm{mg} / \mathrm{dm}^{3}\right)$} & \multicolumn{3}{|c|}{$\left(\mathrm{cmol}_{\mathrm{c}} / \mathrm{dm}^{3}\right)$} & & $(\%)$ \\
\hline \multirow{3}{*}{ Batemans Bay } & $00-10$ & 4,5 & 40 & 5,5 & 181,5 & 2,5 & 1,7 & 1,1 & 5,7 & 35,25 \\
\hline & $10-20$ & 4,6 & 28 & 3,0 & 132,5 & 2,0 & 1,3 & 2,0 & 5,7 & 29,25 \\
\hline & $20-30$ & 4,4 & 24 & 2,0 & 125,5 & 1,0 & 0,7 & 2,4 & 5,2 & 12,50 \\
\hline \multirow{3}{*}{ Bodalla } & $00-10$ & 4,7 & 32 & 5,3 & 110,5 & 2,2 & 1,5 & 0,6 & 4,6 & 43,50 \\
\hline & $10-20$ & 4,5 & 21 & 2,6 & 71,5 & 0,9 & 0,7 & 1,7 & 3,4 & 22,25 \\
\hline & $20-30$ & 4,5 & 16 & 2,0 & 68,5 & 0,5 & 0,4 & 2,5 & 3,5 & 11,75 \\
\hline \multirow{3}{*}{ Lake George } & $00-10$ & 4,2 & 37 & 13,0 & 162,5 & 1,6 & 1,2 & 1,4 & 4,6 & 28,00 \\
\hline & $10-20$ & 4,2 & 23 & 6,0 & 125,0 & 0,9 & 0,5 & 2,5 & 4,3 & 13,50 \\
\hline & $20-30$ & 4,2 & 28 & 3,8 & 103,0 & 0,9 & 0,6 & 2,8 & 5,0 & 9,00 \\
\hline
\end{tabular}


levada ao Laboratório de Ecologia Florestal do Departamento de Ciências Florestais da Universidade Federal de Santa Maria, onde cada componente foi embalado em sacos de papel pardo e colocado em estufa com circulação forçada $\left(75^{\circ} \mathrm{C}\right)$, até atingir peso constante.

A madeira do tronco foi amostrada ao retirar um disco de 5,0 cm de espessura na metade da altura total da árvore (Yong \& Carpenter, 1976), sendo um disco por árvore e de cada procedência. Desse disco, foram separadas a casca e a madeira e foi registrada a massa fresca de cada componente. Após a secagem em estufa, as amostras desses dois componentes foram picadas, para facilitar a moagem. As amostras foram moídas e passadas em peneira com malha de $1,0 \mathrm{~mm}$. De cada amostra moída retirouse uma parte, para realização das análises químicas.

Foram feitas as determinações de N, P, K, Ca, Mg e $\mathrm{S}$ em cada componente da árvore, ou seja, em cada uma das noves árvores por procedência, seguindo as metodologias propostas por Tedesco et al. (1995). As análises foram realizadas no Laboratório de Análise de Solos do Departamento de Solos da Faculdade de Agronomia da Universidade Federal do Rio Grande do Sul. Para realização da determinação de cada nutriente em cada componente da biomassa foram utilizadas três subamostras e, por fim, a média, para realização dos cálculos.

O estoque de macronutrientes contidos em todos os componentes da biomassa acima do solo, em kg/ha, foi obtido a partir da biomassa estimada por Caldeira (1998) e do teor dos macronutrientes em cada componente. A soma dos valores dos macronutrientes para cada componente da biomassa acima do solo forneceu o seu conteúdo total em $\mathrm{kg} / \mathrm{ha}$.

Para estimar o índice de eficiência de utilização dos nutrientes (EUN) para os diferentes componentes das árvores, foi utilizada a fórmula proposta por Hansen \& Baker (1979), em que EUN = kg de matéria seca produzida $\div \mathrm{kg}$ de nutriente utilizado.

Para comparação dos valores médios da EUN de cada componente da biomassa em cada procedência, foi utilizado o delineamento inteiramente casualizado, ao qual foi aplicado o teste de Student Neuman Keul's (SNK), a $5 \%$ probabilidade.

\section{RESULTADOS E DISCUSSÃO}

A eficiência de utilização dos nutrientes para produção de biomassa total acima do solo variou em função da procedência (Quadro 2). A Bodalla foi $18,1 \%$ mais eficiente que a Lake George e 16,2\% mais eficiente que a Batemans Bay na produção de biomassa de todos os componentes das árvores. No entanto, aos 2,4 anos de idade a procedência Bodalla possui biomassa total média menor que a metade da Lake George e Batemans Bay.

Tal fato pode ser atribuído à sua menor tolerância às geadas, que ocorrem com freqüência na região durante o inverno, o que segundo Wang (1982) pode inibir o crescimento das plantas. Searle et al. (1991) observaram que as procedências de acácia-negra de maior altitude são mais tolerantes às geadas, o que parece não ser o caso da Bodalla, pois na sua região de ocorrência natural a altitude é de aproximadamente $15 \mathrm{~m}$.

As três procedências utilizaram mais eficientemente os nutrientes para formação da biomassa acima do solo, na seguinte ordem decrescente: $\mathrm{P}>\mathrm{S}>\mathrm{Mg}>\mathrm{Ca}>\mathrm{K}>$ N. No trabalho realizado por Drumont et al. (1997), foram constatadas algumas semelhanças na ordem de eficiência de utilização dos nutrientes nas procedências analisadas, em dez espécies não-leguminosas nativas da Floresta Atlântica.

As três procedências não apresentaram diferenças significativas na eficiência de uso do $\mathrm{N}$ para produção de todos os componentes da árvore, com exceção da madeira. A procedência Batemans Bay apresentou maior tendência de eficiência de utilização desse elemento para produção de madeira que a Lake George, porém não havendo diferenças significativas. A procedência Bodalla foi a menos eficiente na utilização do $\mathrm{N}$ para produção de madeira. $\mathrm{O} N$ possui eficiência de uso relativamente baixa, quando comparado aos outros nutrientes, devido aos altos teores nas folhas verdes e à retranslocação interna, voltando ao solo através da queda de serapilheira, sendo assim novamente integrado ao ciclo biogeoquímico.

Para produção de folhas e casca, as três procedências não apresentaram diferenças significativas na eficiência de uso do P. A procedência Bodalla foi a mais eficiente na utilização de tal nutriente na produção de galhos vivos e mortos, apesar de existir menor suprimento do elemento nas suas condições de solo (Quadro 2).

A Lake George caracterizou-se como a procedência menos eficiente na utilização do $\mathrm{P}$ para produção de folhas, galhos vivos, casca e madeira, mesmo estando nas melhores condições de solo em se tratando de teores 
Quadro 2 - Biomassa e eficiência nutricional de N, P, K, Ca, Mg e S em três procedências acácia-negra (Acacia mearnsii De Wild.) com 2,4 anos de idade, em Butiá-RS

Table 2 - Biomass and nutritional efficiency of $N, P, K, C a, M g$ and $S$ in three provenances of black wattle (Acacia mearnsii De Wild.), 2.4 years old in Butiá-RS

\begin{tabular}{|c|c|c|c|c|c|c|c|}
\hline \multirow{2}{*}{ Componentes } & Biomassa & $\mathrm{N}$ & $\mathrm{P}$ & $\mathrm{K}$ & $\mathrm{Ca}$ & $\mathrm{Mg}$ & $S$ \\
\hline & $(\mathrm{kg} / \mathrm{ha})$ & \multicolumn{6}{|c|}{$\mathrm{kg}$ de biomassa/kg nutriente utilizado } \\
\hline & \multicolumn{7}{|c|}{ Folhas } \\
\hline Bodalla & $4.376,8$ & $43,2 \mathrm{a}$ & $1.050,9 \mathrm{a}$ & $188,8 \mathrm{~b}$ & $151,3 \mathrm{a}$ & $634,1 \mathrm{a}$ & $874,8 \mathrm{~b}$ \\
\hline Batemans Bay & $7.197,4$ & $43,0 \mathrm{a}$ & 957,9 a & $100,7 \mathrm{a}$ & $208,8 \mathrm{~b}$ & 546,2 a & $804,0 \mathrm{a}$ \\
\hline \multirow[t]{2}{*}{ Lake George } & $7.486,4$ & $42,2 \mathrm{a}$ & $915,7 \mathrm{a}$ & 89,1 a & $236,2 \mathrm{~b}$ & $568,5 \mathrm{a}$ & $742,7 \mathrm{a}$ \\
\hline & \multicolumn{7}{|c|}{ Galhos vivos } \\
\hline Bodalla & $3.850,7$ & $133,1 \mathrm{a}$ & $3.194,3 \mathrm{~b}$ & $170,2 \mathrm{~b}$ & $254,7 \mathrm{a}$ & $1.046,0 \mathrm{a}$ & $1.874,9 \mathrm{~b}$ \\
\hline Batemans Bay & $7.051,7$ & $153,1 \mathrm{a}$ & $2.473,5 \mathrm{ab}$ & $146,0 \mathrm{~b}$ & $331,2 \mathrm{~b}$ & $1.225,3 \mathrm{a}$ & $1.797,8 \mathrm{~b}$ \\
\hline \multirow[t]{2}{*}{ Lake George } & $8.008,2$ & $126,8 \mathrm{a}$ & $1.918,0 \mathrm{a}$ & $107,2 \mathrm{a}$ & $336,7 \mathrm{~b}$ & $1.011,3 \mathrm{a}$ & $1.389,0 \mathrm{a}$ \\
\hline & \multicolumn{7}{|c|}{ Galhos mortos } \\
\hline Bodalla & 75,4 & $232,5 \mathrm{a}$ & $8.888,9 \mathrm{~b}$ & $454,5 \mathrm{~b}$ & $244,3 \mathrm{a}$ & $1.555,5 \mathrm{~b}$ & $4.444,3 \mathrm{~b}$ \\
\hline Batemans Bay & $1.031,0$ & $195,8 \mathrm{a}$ & $2.566,1 \mathrm{a}$ & $309,2 \mathrm{a}$ & $272,4 \mathrm{a}$ & $1.415,8 \mathrm{ab}$ & $3.055,4 \mathrm{a}$ \\
\hline \multirow[t]{2}{*}{ Lake George } & 513,6 & $186,3 \mathrm{a}$ & $7.222,2 \mathrm{~b}$ & $251,1 \mathrm{a}$ & $280,1 \mathrm{a}$ & $943,4 \mathrm{a}$ & $3.425,8 \mathrm{a}$ \\
\hline & \multicolumn{7}{|c|}{ Casca } \\
\hline Bodalla & $2.421,3$ & 89,4 a & $2.388,9 \mathrm{a}$ & $223,5 \mathrm{~b}$ & $166,7 \mathrm{a}$ & $909,3 \mathrm{a}$ & $1.527,9 \mathrm{a}$ \\
\hline Batemans Bay & $4.265,0$ & $100,3 \mathrm{a}$ & $2.407,4 \mathrm{a}$ & $180,5 \mathrm{a}$ & $151,0 \mathrm{a}$ & $826,0 \mathrm{a}$ & $2.113,9 \mathrm{a}$ \\
\hline \multirow[t]{2}{*}{ Lake George } & $4.751,6$ & $98,4 \mathrm{a}$ & $2.277,8 \mathrm{a}$ & $147,2 \mathrm{a}$ & $145,7 \mathrm{a}$ & $728,1 \mathrm{a}$ & $1.714,5 \mathrm{a}$ \\
\hline & \multicolumn{7}{|c|}{ Madeira } \\
\hline Bodalla & $8.751,3$ & $436,9 \mathrm{a}$ & $8.611,1 \mathrm{~b}$ & $333,3 \mathrm{a}$ & $1.441,4 \mathrm{a}$ & $4.351,8 \mathrm{a}$ & $8.888,9 \mathrm{a}$ \\
\hline Batemans Bay & $16.607,6$ & $528,8 \mathrm{~b}$ & $8.888,9 \mathrm{~b}$ & $408,8 \mathrm{a}$ & $1.655,4 \mathrm{a}$ & $4.814,8 \mathrm{a}$ & $7.222,2 \mathrm{a}$ \\
\hline Lake George & $20.346,0$ & $517,2 \mathrm{~b}$ & $4.814,8 \mathrm{a}$ & 359,8 a & $1.574,2 \mathrm{a}$ & $4.351,8 \mathrm{a}$ & $8.333,3 \mathrm{a}$ \\
\hline
\end{tabular}

Médias seguidas pela mesma letra não diferem estatisticamente entre si pelo teste de SNK $(\alpha=0,05)$.

de P (Quadro 2). Na produção de madeira a Batemans Bay e a Bodalla foram as mais eficientes no uso do P, sendo a primeira com maior propensão.

As três procedências apresentaram a maior eficiência no uso do P para produção de biomassa total acima do solo. A procedência Bodalla destacou-se produzindo cerca de 24,1 Mg de biomassa total acima do solo por cada quilo de $\mathrm{P}$, sendo $71,0 \%$ mais eficiente que as procedências Lake George e Batemans Bay. Em Eucalyptus saligna e E. dunnii, Schumacher (1995) também verificou que o $\mathrm{P}$ apresentava a maior eficiência de utilização para produção de biomassa dos vários componentes das árvores. Tal fato é confirmado por Vitousek (1984), que constatou que o P é o único elemento que possui elevada eficiência de utilização nas florestas tropicais, principalmente em áreas onde ele se encontra em baixos teores, como é o presente caso.

As árvores da procedência Bodalla foram as mais eficientes no uso do K para produção de folhas, galhos vivos, galhos mortos e casca, mesmo crescendo em solos com menores teores desse elemento (Quadro 2). Para produção de madeira, as três procedências não apresentaram diferenças significativas quanto à eficiência de uso do $\mathrm{K}$, no entanto a procedência Batemans Bay mostrou ser mais eficiente.

A Lake George foi a procedência menos eficiente no uso do K para produção de folhas, cascas, galhos vivos e mortos. Morais et al. (1990) também verificaram que o Eucalyptus citriodora, plantado em Viçosa-MG, foi pouco eficiente na utilização desse elemento para produção de biomassa acima do solo.

Em se tratando da eficiência do uso de Ca para produção de casca, madeira e galhos mortos, não foram constatadas diferenças significativas entre as procedências. Na produção de folhas e galhos vivos, a Lake George foi a procedência que utilizou o Ca com maior eficiência, sem diferenças significativas quando comparadas com Batemans Bay, em ambos componentes. 
As três procedências de acácia-negra apresentaram eficiência de uso do Ca muito alta, quando comparada com outras leguminosas (Gonçalves et al., 1992). Tal diferença pode ser atribuída a espécie, idade, sítio, disponibilidade de nutriente no solo, teor do nutriente no componente da biomassa e produção de biomassa. Independentemente da procedência, o $\mathrm{Ca}$ foi o elemento menos eficiente para produção de casca, em relação aos demais componentes da biomassa. Tal fato talvez ocorra em virtude do elevado teor de Ca na casca, o que pode ser atribuído, em parte, à grande imobilização desse elemento no floema, principalmente na forma de oxalato de cálcio.

Em relação à eficiência de utilização do $\mathrm{Mg}$ para produção de folhas, casca, galhos vivos e madeira, as três procedências não apresentaram diferenças significativas, porém a Bodalla teve destaque para os dois primeiros componentes e a Batemans Bay, para os dois últimos. Nas folhas e na casca das procedências estudadas, a eficiência do uso de $\mathrm{Mg}$ foi menor em relação à dos demais componentes. Tal observação também foi verificada por Schumacher (1995), em Eucalyptus globulus, E. bicostata, E. maidenii e E. saligna, com 4 anos de idade.

Não foram constatadas diferenças significativas entre as procedências na eficiência do uso de $\mathrm{S}$ para produção de casca e madeira, porém a Batemans Bay e a Bodalla foram as mais eficientes na utilização de $\mathrm{S}$ para produção de ambos os componentes. A Bodalla foi a procedência com maior eficiência no uso de $\mathrm{S}$ para produzir biomassa de folhas, galhos vivos e mortos (copa). Depois do P, o S foi o nutriente mais eficientemente utilizado pelas árvores das três procedências para produção de biomassa, principalmente na produção de madeira.

Levando em consideração a produção de fuste (madeira e casca) e copa, as procedências Bodalla e Batemans Bay apresentaram maior eficiência de uso em todos os nutrientes. A Lake George apresentou menor eficiência de uso dos nutrientes estudados para produção de todos os componentes, exceto galhos mortos, o que pode ser atribuído aos maiores teores dos elementos minerais na biomassa (Caldeira, 1998).

\section{CONCLUSÕES}

A procedência Bodalla foi a que melhor utilizou os nutrientes para produção de biomassa total da copa, tendo sido 34,7 e 22,2\% mais eficiente que a Batemans Bay e Lake George, respectivamente. Para produção de fuste, essa procedência foi 14,7\% mais eficiente no uso de todos os nutrientes em relação a Lake George e praticamente sem diferença entre a Batemans Bay.

Apesar de a Bodalla ser a procedência mais eficiente na utilização dos nutrientes para produção de copa e fuste, ela não tolera geada. Em contrapartida, a Lake George tolera geadas e tem grande capacidade produtiva de biomassa total acima do solo, mas é a procedência menos eficiente no uso dos nutrientes para formação de copa e fuste.

Em solos bastante intemperizados e pobres em bases, onde se encontra baixo teor de $\mathrm{P}$, a procedência Bodalla poderá ter melhor desempenho, pois apresenta grande eficiência no uso desse nutriente para produzir biomassa, principalmente madeira.

\section{REFERÊNCIAS BIBLIOGRÁFICAS}

CALDEIRA, M. V. W. Quantificação da biomassa e do conteúdo de nutrientes em diferentes procedências de acácia-negra (Acacia mearnsii De Wild.). Santa Maria: Universidade Federal de Santa Maria, 1998. 96 p. Dissertação (Mestrado em Engenharia Florestal) Universidade Federal de Santa Maria, 1998.

DALLAGO, J. S. Utilização da cinza de biomassa de caldeira como fonte de nutrientes no crescimento de plantas de acácia-negra (Acacia mearnsii De Wild.). Santa Maria: Universidade Federal de Santa Maria, 2000. 64 p. Dissertação (Mestrado em Engenharia Florestal) Universidade Federal de Santa Maria, 2000.

DRUMOND, M. A. et al. Composição mineral e demanda nutricional de espécies florestais da Mata Atlântica. Revista Árvore, v. 21, n. 1, p. 1-10, 1997.

EMPRESA BRASILEIRA DE PESQUISA

AGROPECUÁRIA - EMBRAPA. Sistema brasileiro de classificação levantamento de solos. Rio de Janeiro: 1999. 212 p.

GONÇALVES, J. L. M. et al. Capacidade de absorção e eficiência nutricional de algumas espécies arbóreas tropicais. In: CONGRESSO NACIONAL SOBRE ESSÊNCIAS NATIVAS, 2., São Paulo. Revista do Instituto Florestal, v. 4, p. 463-469, 1992.

HANSEN, E. A.; BAKER, J. B. Biomass and nutrient renewal in short rotation intensively cultured plantations. In: ANNUAL MEETING AMERICAM POPLARS COUNCIL, 1979, Thompsonville. Annual... Thompsonville: 1979. p. 130-151. 
HIGA, A. R. et al. Desarrollo de sistemas de produccoón para acacia negra (Acacia mearnsii De Wild.). In: CONGRESSO LATINOAMERICANO IUFRO - O manejo sustentable de los recursos forestales, desafío del siglo XXI, 1., 1998, Valdivia. Congresso... Valdivia: IUFRO, 1998.

INSTITUTO DE PESQUISAS AGRONÔMICAS IPAGRO. Atlas agroclimático do Estado do Rio Grande do Sul. Porto Alegre: 1989. 3 v.

MORAIS, E. J. et al. Biomassa e eficiência nutricional de espécies de eucalipto em duas regiões bioclimáticas de Minas gerais. Revista Brasileira de Ciência do Solo, v. 14, p. $353-362,1990$.

MORENO, J. A. Clima do Rio Grande do Sul. Porto Alegre: Secretaria da Agricultura, 1961. 42 p.

SCHUMACHER, M. V. Naehrstoffkreislauf in verschiedenen Bestaeden von Eucalyptus saligna (Smith), Eucalyptus dunnii (Maiden) und Eucalyptus globulus (Labillardière) in Rio Grande do Sul, Brasilien. Wien: Universitäet für Bodenkultur, 1995. 167 p. Tese

(Doutorado em Ecologia e Nutrição Florestal) - Universitäet für Bodenkultur, 1995.
SEARLE, S. D. et al. Advances in tropical research. In: INTERNATIONAL WORKSHOP HELD IN BANGKOK, THAILAND, 1991, Thailand. Proceedings... Canberra: ACIAR, 1991. p. 93-94.

TEDESCO, M. J. et al. Análise de solos, plantas e outros materiais. Porto Alegre: Universidade Federal do Rio Grande do Sul, 1995. 174 p. (Boletim Técnico, 5).

VITOUSEK, P. M. Litterfall, nutrient cycling and nutrient limitation in tropical forests. Ecology, v. 65, p. 285-298, 1984.

WANG, C. Y. Physiological and biochemical responses of plants to chilling stress. Hort Science, v. 17, p. 173-186, 1982.

YONG, H. E.; CARPENTER, P. N. Sampling variation of nutrient element content within and between trees of the same species. In: OSLO BIOMASS STUDIES, 1976, Oslo. Proceedings... Oslo: 1976. p. 75-90. 